Tropical Journal of Pharmaceutical Research, June 2006; 5 (1): 533-538

(C) Pharmacotherapy Group, Faculty of Pharmacy, University of Benin Benin City, Nigeria

All rights reserved.

Research Article

Available online at http://www.tjpr.org

\title{
In-Vitro Adsorption of Fluoroquinolones on Some Pharmaceutical Adsorbents
}

\author{
Chukwuenweniwe J. Eboka* and Aderemi B. Afolabi
}

Department of Pharmaceutical Chemistry, University of Benin, Benin City.

\begin{abstract}
Purpose: Drug overdose and poisoning are common clinical problems and could occur with the fluoroquinolones - a new series of synthetic antimicrobial agents. It therefore becomes important to study the adsorption of the fluoroquinolones on pharmaceutical adsorbents which could serve as possible antidotes for the emergency treatment of fluoroquinolone overdose or poisoning when they occur.

Method: The rate and extent of adsorption of the fluoroquinolones on some pharmaceutical adsorbents, namely activated charcoal, kaolin and bentonite were investigated spectrophotometrically

Results: The fluoroquinolones adsorbed on activated charcoal rapidly and attained equilibrium within fifteen minutes. The fluoroquinolones however adsorbed on kaolin and bentonite less rapidly and attained equilibrium within two hours. Activated charcoal and bentonite had high adsorption capacities for the fluoroquinolones while kaolin had low adsorption capacities for them.

Conclusion: Because of the rapid rate of adsorption and high binding capacities exhibited by activated charcoal for the fluoroquinolones, it could be an effective antidote for the fluoroquinolones in cases of overdose or poisoning. Activated charcoal has shown a superior behaviour to both bentonite and kaolin in the adsorption of the fluoroquinolones.
\end{abstract}

${ }^{\star}$ Corresponding Author: E-mail: chukeboka@yahoo.com 


\section{INTRODUCTION}

Drug poisoning has been defined as a condition produced by any substance which when swallowed, inhaled, injected or absorbed percutaneously is capable of causing death, injury, toxic or untoward reactions ${ }^{1}$. Poisoning is a very common clinical problem and could occur with the fluoroquinolones. The major principles applied in the emergency treatment of accidental poisoning by drugs are dilution, emesis and adsorption $^{2}$. In cases where no specific antidotes exist, prevention of further absorption of a drug from the oral route is by use of oral adsorbents. This could be of immense benefit in the management of drug over dose and/or poisoning. The use of standard adsorbents such as activated charcoal in the prevention of further absorption of drugs, is recognised in clinical practice $^{3}$. The safety of activated charcoal is remarkable because it is non reactive and non absorbable from the gastro-intestinal tract ${ }^{4}$.

The fluoroquinolones are a new series of synthetic antimicrobial agents that have a broad spectrum of activity against both gram-positive and gram-negative organisms ${ }^{5,6}$. They are available and active as oral dosage forms. They are well absorbed from the oral route and distribute well into body fluids and tissues to attain effective inhibitory concentrations ${ }^{7-9}$. Though they are well tolerated, a few adverse effects which include gastrointestinal effects, disturbances of the CNS, phototoxic reactions, liver enzyme abnormalities, arthropathy, tendinitis and tendon rupture and prolongation of $\mathrm{QT}_{\mathrm{c}}$ interval have been reported ${ }^{10-13}$. Though no reports are available on accidental or deliberate poisoning with the fluoroquinolones, their extensive use warrants investigation into possible effective antidotes in cases of accidental poisoning or overdose especially in elderly patients, in patients with renal impairment, and in children in whom these fluoroquinolones are contra-indicated as a result of drug-induced arthropathy. We present in this report our investigations into the in-vitro adsorption of the fluoroquinolones exemplified by ciprofloxacin, ofloxacin and norfloxacin (the most commonly prescribed and available fluoroquinolones) on some commercially available pharmaceutical adsorbents - activated charcoal, kaolin and bentonite. The aim is to provide baseline data that would characterise the rate and extent of adsorption of the fluoroquinolones on these adsorbents which could guide the pharmacist and other healthcare givers in the choice of antidotes in the event of overdosage and/or poisoning with the fluoroquinolones.

\section{EXPERIMENTAL \\ Materials}

Ofloxacin powder was kindly supplied by Hoechst Germany, through Nigeria - German Chemicals, Nigeria. Pure powders of ciprofloxacin hydrochloride and norfloxacin were supplied by Sam Pharmaceuticals, Nigeria. Adsorbents used included activated charcoal and light kaolin from BDH Chemicals, UK, while bentonite was from Halewood Chemicals Ltd, UK. Hydrochloric acid was from Sigma - Aldrich Laboratory, Germany.

Equipment: Ultrospec 3000 ultra-violet/visible spectrophotometer from Pharmacia Biotech, UK was used to take all the absorbance readings. Thermo- statted shaker bath from Gallenkamp, UK set at $37 \pm 0.2^{\circ} \mathrm{C}$ was used to shake fluoroquinolone - adsorbent mixtures and $B \alpha T$ hot air oven from Bird and Tat lock UK was used to dry the adsorbents.

\section{Methods}

Standard solutions of each of the fluoroquinolones were made in $0.1 \mathrm{M}$ hydrochloric acid. The absorbances of each of the fluoroquinolone solutions were measured at their respective wavelengths of maximum absorption and their respective Beer's plots made.

The adsorbents were activated by drying at $110^{\circ} \mathrm{C}$ for five hours in the oven for activated charcoal and for three hours for kaolin and bentonite. They were then cooled and stored in the descicator. Two types of experiments were carried out. The first was to investigate the time to attain equilibrium while the second was to find out the extent of adsorption of each of the 


\section{Eboka et al}

fluoroquinolones on the adsorbents. For the time to reach equilibrium study, $15 \mathrm{ml}$ of standard solutions of each fluoroquinolone in $0.1 \mathrm{M}$ hydrochloric acid were added separately into $25 \mathrm{ml}$ volumetric flasks to which graded amounts of each adsorbent were put. Similarly, $15 \mathrm{ml}$ of $0.1 \mathrm{M}$ hydrochloric acid was added to equal graded amounts of adsorbent in $25 \mathrm{ml}$ volumetric flasks. The slurries were mixed and put in the shaker bath set at 80 oscillations per minute and at $37 \pm 0.2^{\circ} \mathrm{C}$. Aliquots of the mixtures were withdrawn at separate time intervals and centrifuged at $3000 \mathrm{~g}$ for five minutes. Portions of the clear supernatant was diluted serially and the absorbance of the final solution measured at the $\lambda_{\max }$ for the respective drug. The slurries with $15 \mathrm{ml} 0.1 \mathrm{M}$ hydrochloric acid (without fluoroquinolone) treated in a similar way served as blanks for the absorbance measurements. From the Beer's plot for the fluoroquinolone previously made, the amount of free drug in solution was determined. From the results, the time to attain equilibrium for each adsorbent was determined. The procedure was repeated for each pair of fluoroquinolone and adsorbent.

For the adsorption equilibrium studies, $15 \mathrm{ml}$ of standard solution of fluoroquinolone was added to graded amounts of adsorbent in separate $25 \mathrm{ml}$ volumetric flasks. Similarly, $15 \mathrm{ml}$ of $0.1 \mathrm{M}$ hydrochloric acid was added to equal graded amounts of adsorbent in $25 \mathrm{ml}$ volumetric flasks. The slurries were mixed and put in a shaker bath set at 80 oscillations per minute and at $37 \pm 0.2^{\circ} \mathrm{C}$. The shaking was continued for a period exceeding the time to attain equilibrium for the adsorbent. Thereafter, part of the mixture was taken and treated as above. Aliquot of the clean supernatant was diluted serially and the absorbance of the resultant fluoroquinolone solution measured at its $\lambda_{\max }$. The slurries with $15 \mathrm{ml} \quad 0.1 \mathrm{M}$ hydrochloric acid (without fluoroquinolone) treated in a similar way served as blanks for the absorbance measurements. From the Beer's plot of the fluoroquinolone obtained earlier at the $\lambda_{\max }$, the amounts of free drug remaining in solution at equilibrium in the presence of graded amounts of the adsorbent were determined hence the amount adsorbed was calculated. The procedure was repeated for each fluoroquinolone - adsorbent pair.

\section{RESULTS}

In $0.1 \mathrm{M}$ hydrochloric acid, ciprofloxacin, ofloxacin and norfloxacin had $\lambda_{\max }$ values of $277 \mathrm{~nm}, 278 \mathrm{~nm}$ and $274 \mathrm{~nm}$ respectively. At each $\lambda_{\max }$, Beer's law was obeyed for each fluoroquinolone. The study to know when equilibrium was attained showed that with activated charcoal as adsorbent, adsorption equilibrium was attained rapidly within 15 minutes with each of the fluoroquinolones. But with kaolin and bentonite, adsorption equilibrium was attained less rapidly but within two hours with each of the fluoroquinolones. In the presence of graded amounts of each adsorbent, the equilibrium studies fitted into Langmuir's equation $^{14}$.

$$
\frac{C_{e q}}{x / m}=\frac{C_{e q}}{k_{2}}+\frac{1}{k_{1} k_{2}}
$$

Hence;

$$
\frac{1}{x / m}=\frac{1}{k_{2}}+\frac{1}{k_{1} k_{2}} \times \frac{1}{C_{e q}}
$$

Figure 1 shows a typical Langmuir isotherm plot obtained from the adsorption studies. The results of the binding constants obtained for the different fluoroquinolones with the different adsorbents are shown in Table 1.

\section{DISCUSSION}

$0.1 \mathrm{M}$ hydrochloric acid was used to mimic the acidity of the stomach where drugs that are taken orally are retained for some time prior to absorption from the gastro-intestinal tract. Shaking at $37^{\circ} \mathrm{C}$ was to mimic gastro-intestinal motility and body temperature. From the time to attain equilibrium studies, activated charcoal adsorbed the fluoroquinolones rapidly thus equilibrium was attained within 15 minutes. This is a desirable feature of an adsorbent antidote. The ability to adsorb any poison or excess drug rapidly in the case of overdose helps to prevent the poison or drug from being absorbed significantly and thus reduces fatality and mortality. Activated charcoal has been known to have this desirable property, hence it has been used generally as an antidote ${ }^{15-17}$. On the other hand, kaolin and bentonite were slower to attain equilibrium with the fluoroquinolones. It took 
Table 1: Values of Langmuir adsorption constants for the fluoroquinolones on pharmaceutical adsorbents

\begin{tabular}{|l|c|c|c|}
\hline Fluoroquinolone & Adsorbent & $\mathbf{K}_{\mathbf{2}}^{\mathbf{a}}$ & $\mathbf{K}_{\mathbf{1}}$ \\
\hline Ciprofloxacin & AC & 13 \\
Norfloxacin & AC & 206 & 16 \\
Ofloxacin & AC & 302 & 4 \\
Ciprofloxacin & Bentonite & 171 & 52 \\
Norfloxacin & Bentonite & 153 & 267 \\
Ofloxacin & Bentonite & 95 & 350 \\
Ciprofloxacin & Kaolin & 9 & 4 \\
Norfloxacin & Kaolin & 6 & 16 \\
Ofloxacin & Kaolin & 2 & 57 \\
\hline
\end{tabular}

a Expressed as milligram of drug per gram of adsorbent.

$b \quad A C$ is activated charcoal

about two hours to attain equilibrium. This is a long time within which much of the drugs would have been absorbed to cause toxic effects in the case of overdose or poisoning because the fluoroquinolones are rapidly absorbed from the oral route ${ }^{18}$.

The binding constants on the adsorbents were calculated from the Langmuir isotherms. The results showed that the fluoroquinolones had a strong binding to activated charcoal. The values obtained here for the binding constants $\left(\mathrm{k}_{2}\right)$ of the fluoroquinolones on activated charcoal are in the same range of values as the those obtained for some other drugs by other workers. Sellers et $a^{14}$ obtained the binding constant of aspirin, gluthetimide, amitriptyline, chlordiazepoxide, diazepam, methaqualone on activated charcoal at $37^{\circ} \mathrm{C}$ and in similar acidic condition as 262 , 252, 133, 157, 136 and 179 respectively. Thus, activated charcoal can bind the fluoroquinolones quickly and strongly and prevent them from being absorbed from the acidic medium of the stomach. The recommended practice to prevent vomiting induced by large amounts of activated charcoal is to administer $1 \mathrm{~g}$ of charcoal per kilogram of body weight. If this recommendation is followed, very little of any of the fluoroquinolones would be available for absorption following activated charcoal administration. The binding constants of bentonite for the fluoroquinolones were also high and of the same order of magnitude as those of activated charcoal. Thus, it can be an effective antidote for fluoroquinolone overdose. Bentonite has been shown to have a stronger adsorption capacity for aromatic hydrocarbons than kaolin ${ }^{19}$. Though both bentonite and activated charcoal had similar binding constants, the rate of adsorption on bentonite was slower and thus activated charcoal would be preferred to bentonite. For all the fluoroquinolones, the binding constants for kaolin was very low. Thus, kaolin cannot effectively bind the fluoroquinolones and prevent them from being absorbed from the gastro-intestinal tract in the case of overdose or accidental poisoning. Kaolin cannot be an effective antidote for the fluoroquinolones.

The mechanism of adsorption of various drugs on activated charcoal has been extensively studied and known to proceed by surface phenomenon ${ }^{20}$. The high binding capacity of activated charcoal has been attributed to its high surface area. Similarly, kaolin is an adsorbent 


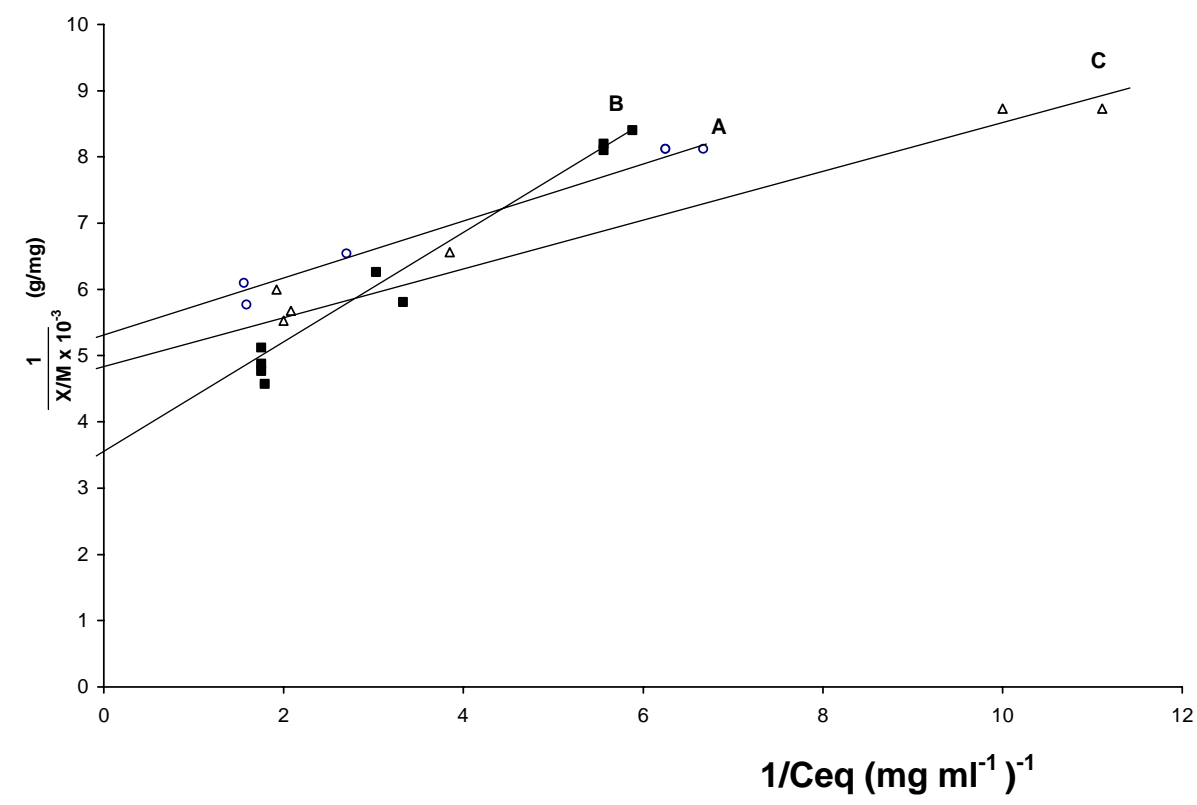

Fig. 1: Adsorption of Norfloxacin (A), Ofloxacin (B), and Ciprofloxacin (C) on Activated Charcoal

that is normally administered in the symptomatic treatment of enteritis, colitis and dysentery associated with food and alkaloidal poisoning. Adsorption of drugs on it is also purely a surface phenomenon. But for bentonite which swells in aqueous medium to form a gel, the mechanism of adsorption may be by entrapment of the fluoroquinolones by the gel rather than by surface adsorption. In the acidic medium used in this study, the fluoroquinolones existed in their cationic forms. Thus when adsorbed on the surface of the adsorbents they would repel each other and incoming cations electrostatically, hence they cannot pack densely onto the surface. This may be the reason why Langmuir's isotherm was followed as multi-layer adsorption of drugs may not be possible. Despite such limitations, activated charcoal still adsorbed the fluoroquinolones strongly and rapidly.

Kaolin and bentonite were chosen for this study because of their reported adsorptive properties with other drugs. They are also commonly used as drugs and in pharmaceutical formulations. They are safe. But more importantly, they were investigated as alternatives to activated charcoal which precipitates vomiting when large doses are given. From the results, it has become obvious that the adsorptive capacity and rate of adsorption of the fluoroquinolones onto activated charcoal were superior to kaolin. Also, the rate of adsorption onto activated charcoal made it a better antidote for the fluoroquinolones than bentonite. Moreover, the swelling observed with aqueous bentonite solutions will lead to serious constipation unless it is co-administered with a carthartic. Magnesium trisilicate - a known adsorbent was not investigated in this study because magnesium complexes with the fluoroquinolones and the effect due to this complexation will further complicate its adsorptive properties.

\section{CONCLUSION}

Activated charcoal strongly and rapidly adsorbed the fluoroquinolones in acid medium in-vitro. Thus, it could be effectively used to prevent 


\section{Eboka et al}

fluoroquinolone absorption from the gastrointestinal tract in cases of overdose or poisoning. Activated charcoal was superior to both kaolin and bentonite as an adsorbent for the fluoroquinolones. Though bentonite had a high adsorbing capacity for the fluoroquinolones, it was slow in adsorbing them. Moreover, its swelling behaviour in aqueous medium makes it less attractive. Kaolin adsorbed the fluoroquinolones poorly in acid medium. Activated charcoal can serve as a good antidote for fluoroquinolone overdose or poisoning. It should not therefore be administered concurrently with the fluoroquinolones in the treatment of infection as this would lead to therapeutic failure. On the other hand, kaolin can be concomitantly administered with the fluoroquinolones without adversely affecting the therapeutic efficacy of the fluoroquinolones.

\section{References}

1 Plaxo JM. "Poison Control". In: Dispensing of Medication 97th Edition. Eastern Pennsylvania; Merck Publishing Company, 1971; 387.

2 Von - Ottingmen WF. Recent Research in Emergency Treatment of Accidental Poisoning. In: A Guide to Clinical Diagnosis and Treatment. Philadelphia; WB Sandos Company, 1983; 400.

3 Orisakwe OE, Oluboyo A, Ofoefule S, Obi E, Ilondu N, Afonne J, Agbasi $P$ and Chiroma DH. Adsorption studies of artesunate: evaluation of saline carthartics as additive in management of artesunate poisoning. J. Health Sc: 2001; 47(5): $491-494$.

4 Agbasi PU, Orisakwe OF, Ofoefule SI, Ilondu NA and Afonne OJ. Effect of sulphate carthartics on adsorption of rifampicin to activated charcoal. Indian J. Pharm. Sci., 2001; 328 - 330.

5 King $A$ and lan $P$. The comparative in-vitro activity of eight newer quinolones and nalidixic acid. $\mathrm{J}$. Antimicrob. Chemother, 1986; 18(Suppl. D): 1 20.

6 Janknegt $R$. Fluorinated quinolones. A review of the mode of action, antimicrobial activity, pharmacokinetics and clinical efficacy. Pharmaceutisch Weekbl (Sci). 1996; 8: 1 - 21.

7 Adhami ZN, Wise R, Weston $D$ and Crump $B$. The pharmacokinetics and tissue penetration of norfloxacin. J. Antimicrob. Chemother., 1984; 13: $87-92$.
8 Lockley MR, Wise $R$ and Dent J. The pharmacokinetics and tissue penetration of ofloxacin. J. Antimicrob. Chemother; 1984; 14: 647-652.

9 Bergan T, Dalhoff $A$ and Thorsteinsson SB. A review of the pharmacokinetics and tissue penetration of ciprofloxacin. In: Ciprofloxacin. A new 4 quinolone. Findlay R. ed., Sieber and McIntyre, Hong Kong, 1985; 23 - 26.

10 Christie MJ, Nong K, Ting RH, Tam PY and Sikaneta TG. Generalized seizure and toxic epidermal necrolysis following levofloxacin exposure. Ann. Pharmacother., 2005, 39(5): 953 - 955.

11 Dawe RS, Ibbotson SH, Sanderson JB, Thomson EM and Ferguson J. A randomized controlled trial (volunteer study) of ritafloxacin, enoxacin, levofloxacin and sparfloxacin phototoxicity. Br. J. Dermatol., 2003, 149(6); 1232 - 1241.

12 Stahlmann $R$ and Lode $H$. Toxicity of quinolones. Drugs, 1999; 58, Suppl 2: $37-42$.

13. Fish DN. Fluoroquinolone adverse effects and drug interactions. Pharmacotherapy, 2001; 21(10s): 253s - 272s.

14 Sellers EM, Khouw $V$ and Dolman L. Comparative drug adsorption by activated charcoal.J.Pharm. Sci., 1977; 66(11): 1641-1642

15 Becker WJ, Shall RA, Corby DG, Combs HF and Payne $N$. Inhibition of aspirin absorption by activated charcoal and apomorphine. Clin. Pharmac. Ther, 1969; 10: 710.

16 Levy G. Gastrointestinal clearance of drugs with activated charcoal. New Engl. J. Med., 1982; 387: $676-678$.

17 Orisakwe OE, Dioka EC, Orish CN and Ofoefule SI. Effect of activated charcoal on rifampicin absorption in man. Total Exp. Clin. Med., 1995; 21:51- 54

18 Lode H, Hoffken G, Boeckk M, Depperman N, Borner K and Koeppe P. Quinolone pharmacokinetics and metabolism. J. Antimocrob. Chemother., 1990; 26(Suppl. B): $41-49$.

19 EL-Dib MA, Mursy AS and Badawy MI. Role of adsorbents in the removal of soluble aromatic hydrocarbons from drinking water. Water Research, 1978; 12: 1131 - 1137.

20 Cassidy HG. Adsorption and Chromatography. In: Technique of Organic Chemistry. Vol. 5 Weissberger ed. Interscience, New York, 1951; 1049. 